\title{
Semaphorin Signaling in GnRH Neurons: From Development to Disease
}

\author{
Roberto Oleari Antonella Lettieri Alyssa Paganoni Luca Zanieri \\ Anna Cariboni \\ Department of Pharmacological and Biomolecular Sciences, University of Milan, Milan, Italy
}

\section{Keywords}

GnRH deficiency · Neuron development · Semaphorin

\begin{abstract}
In mammals, fertility critically depends on the pulsatile secretion of gonadotropin-releasing hormone $(\mathrm{GnRH})$ by scattered hypothalamic neurons (GnRH neurons). During development, GnRH neurons originate in the nasal placode and migrate first into the nasal compartment and then through the nasal/forebrain junction, before they reach their final position in the hypothalamus. This neurodevelopmental process, which has been extensively studied in mouse models, is regulated by a plethora of factors that might control GnRH neuron migration or survival as well as the fasciculation/targeting of the olfactory/vomeronasal axons along which the $\mathrm{GnRH}$ neurons migrate. Defects in GnRH neuron development or release can lead to isolated $\mathrm{GnRH}$ deficiency, with the underlying genetic causes still being partially unknown. Recently, semaphorins and their receptors neuropilins and plexins, a large family of molecules implicated in neuronal development and plasticity, are emerging as key regulators of GnRH neuron biology and deficiency. Specifically, semaphorins have been shown to play different roles in $\mathrm{GnRH}$ neuron biology by regulating migration and survival during embryonic development as well as secretion in adulthood.
\end{abstract}

(c) 2018 S. Karger AG, Basel

\section{KARGER}

๑ 2018 S. Karger AG, Basel

E-Mail karger@karger.com

www.karger.com/nen

\section{Gonadotropin-Releasing Hormone Neurons}

Gonadotropin-releasing hormone ( $\mathrm{GnRH}$ ) neurons are key neuroendocrine cells in the establishment and control of the hypothalamic-pituitary-gonadal axis. In mammals, GnRH neurons mainly reside in the adult hypothalamus of the brain, where they synthetize and secrete the neurohormone $\mathrm{GnRH}$ in a pulsatile manner. The neurohormone is released at the level of the median eminence (ME) into a system of portal blood vessels to reach the pituitary gland where it stimulates the release of the gonadotropins LH and FSH. Via the general circulation, in turn, FSH and LH reach the gonads where they act directly and stimulate the synthesis of sex steroids (androgens and estrogens), which represent the main hormones involved in sexual maturation and in the regulation of fertility [1].

Unusual aspects characterize the GnRH neuronal system. First, despite their central role in controlling the reproductive axis, GnRH neurons are relatively few and scattered in the brain, where their cell bodies can be found between the olfactory bulbs and the medial preoptic area (MPOA). Second, GnRH neuron development is unique as they originate outside the brain and need to migrate for long distances and through different environments before reaching the brain. This peculiar development has 
been extensively studied in mice, where GnRH neurons are first detected in the nasal placode (NP) around embryonic day (E) 10.5 [2] and then migrate through the nasal compartment into the basal forebrain before continuing their migration within the brain to attain their final positions in the hypothalamus [3, 4]. Recent lineage tracing studies on tissue-specific conditional mouse lines have helped to clarify their embryonic origin, which is now considered to be of placodal and neural crest mixed derivation [5].

Ex vivo nasal explants from embryonic mouse tissues have helped to better clarify some of the features of migrating $\mathrm{GnRH}$ neurons, including their saltatory movement [6] and their axonophilic migration [7]. Thus, $\mathrm{GnRH}$ neurons migrate throughout the nasal mesenchyme along a neural scaffold composed of axons originating from cell bodies that reside in the developing nose. According to the current working model, the axonal scaffold followed by GnRH neurons is formed by intermingled axons extending from olfactory/vomeronasal (OLF/ $\mathrm{VN})$ neuron soma. These neurons are located in the NPderived olfactory epithelium and vomeronasal organ in the nose and project to the main and accessory bulbs, respectively. Then, at the junction between the nose and forebrain, GnRH neurons enter the basal forebrain following a subset of peripherin-expressing axons that turn caudally and that are known as the caudal branch of the vomeronasal nerve (cVNN) [8]. Recently, Taroc et al. [9] demonstrated that $\mathrm{GnRH}$ neuron migration is independent of the correct formation of OLF/VN nerve scaffolds, since arhinencephalic Arx1-null mice displayed normal $\mathrm{GnRH}$ neuron development. Instead, they showed that GnRH neurons appear to migrate along the axons of the putative terminal nerve (TN) or cranial nerve 0 to access the brain. These novel findings are changing the previous view rendering the nature of the axonal scaffold followed by GnRH neurons under debate.

Recently, the study of GnRH neuron migration has been extended to humans thanks to the availability of human fetuses and tissue-clearing technologies, providing evidence that GnRH neuron development is conserved between rodents and primates [10].

\section{Isolated GnRH Deficiency}

Defects in GnRH neuron development or function can lead to a pathological condition known as isolated $\mathrm{GnRH}$ deficiency (IGD). IGD is characterized by low serum concentrations of $\mathrm{LH}$ and $\mathrm{FSH}$ in the presence of low circu- lating concentrations of sex steroids that lead to the absence of puberty and, if not treated, to reproductive failure [11].

Clinically, IGD could be present solely as GnRH deficiency, in association with absent (anosmia) or diminished (hyposmia) sense of smell, or in multisystemic syndromes. When associated with hyposmia/anosmia, IGD is referred to as the Kallmann syndrome (KS) and represents $50 \%$ of overall IGD cases. Instead, when the sense of smell is intact, IGD is referred to as normosmic hypogonadotropic hypogonadism (nHH). Finally, IGD may be present as a clinical trait in multisystemic syndromes or may overlap with multisystemic syndromes sharing only some clinical features, such as the CHARGE syndrome, which is sharing olfactory and reproductive defects with IGD [12-14].

IGD is genetically heterogeneous, with both sporadic and familial cases. Several modes of inheritance have been identified, including X-chromosome-linked recessive, autosomal-recessive, and dominant inheritance [13]. However, IGD is not solely a monogenic disorder as several IGD genes are shown to genetically interact modulating the severity of GnRH deficiency, strengthening the idea that IGD can also be considered a digenic/oligogenic disorder $[15,16]$. To date, up to 30 genes have been demonstrated to lead to IGD, but they account only for the $50 \%$ of overall IGD patients; genetic causes for the remaining cases are so far still unknown [13]. IGD causative genes can be divided into two groups as they disrupt either the development of GnRH neurons (neurodevelopmental genes) or the action/signaling of $\mathrm{GnRH}$ in normally developed GnRH neurons (neuroendocrine genes). Mutations in neurodevelopmental genes usually lead to KS, whereas defective neuroendocrine genes usually underlie $\mathrm{nHH}$. Several genes have a dual role both during development and function of GnRH neurons and, therefore, can cause either $\mathrm{KS}$ or $\mathrm{nHH}$. In addition, some genes have a pleiotropic role, controlling many different developmental processes, so mutations lying on these genes can lead to either $\mathrm{KS} / \mathrm{nHH}$ or syndromic IGD, depending on the nature of the mutation $[13,17]$.

The high degree of phenotypic and genetic variability, in addition to the low penetrance observed for some genes, makes the identification of novel genes implicated in the pathogenesis of IGD difficult. In addition, canonical genetic investigations in affected families are limited by the negative effect of IGD on fertility so that screened patients are few in numbers; finally, our biological knowledge on the hypothalamic-pituitary-gonadal axis is still limited. However, in recent years, several new IGD causative genes have been discovered following two main ap- 


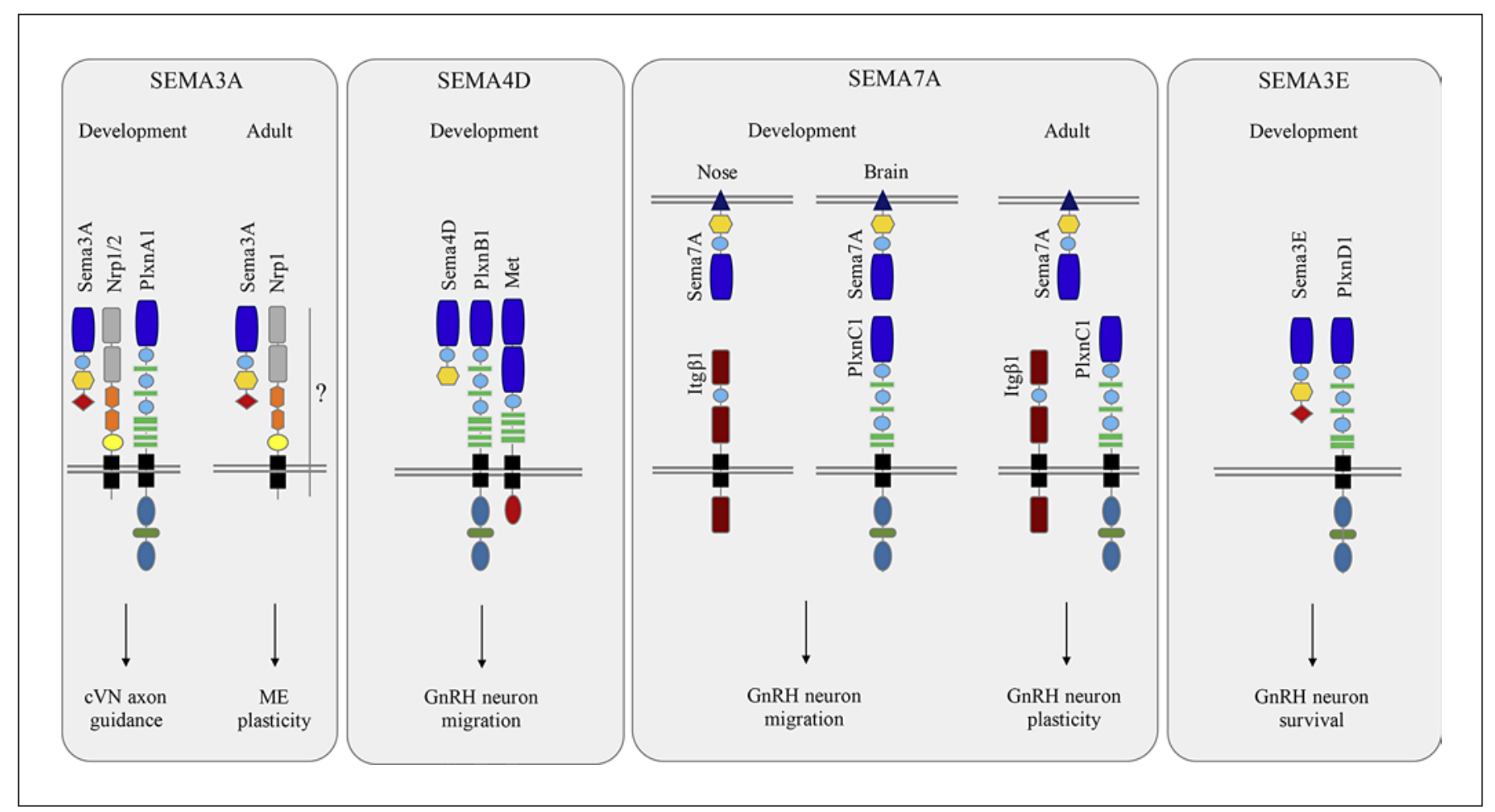

Fig. 1. Schematic drawing showing ligand-receptor complexes and related biological activity of the identified semaphorin signaling pathways in GnRH neuron biology. ME, median eminence; cVN, caudal vomeronasal.

proaches: (i) exome sequencing-based identification of novel mutated genes in affected individuals and functional validation with in silico, in vitro, and/or in vivo experimental models; (ii) basic science-driven identification of candidate genes involved in GnRH neuron physiology and targeted sequencing of the candidate genes in affected individuals $[15,17]$.

\section{Role of Semaphorins and Their Plexin/Neuropilin Receptors in the GnRH Neuronal System}

Over the past few years, work from several laboratories including ours have highlighted the importance of semaphorins and their receptors/coreceptors in the control of the GnRH neuronal system and predicated or validated mutations of such genes in human patients by using different experimental approaches [18-20]. To date, mutations in 3 semaphorins (SEMA3A, SEMA3E, SEMA7A) and in their receptors (PLXNA1, NRP1, NRP2) have been found in IGD patients [19-23] strongly supporting the involvement of these genetic pathways in the control of GnRH neuron physiology.

Semaphorin Signaling in GnRH Neurons:

From Development to Disease
In this minireview, we will examine what is known for each molecule identified from a biological and genetic/ clinical point of view. The roles of semaphorins and their receptors in $\mathrm{GnRH}$ neuron biology are depicted in the schematic drawing in Figure 1.

Semaphorins constitute one of the largest families of guidance cues, are highly conserved across species, and grouped into 8 classes. Semaphorins were first discovered as growth cone collapsing factors and, therefore, as repulsive neuronal guidance cues, but depending on their receptors they can also promote chemoattraction. The expression patterns of semaphorins have been best characterized in the nervous system, where they are dynamically expressed in specific areas during development, while their expression often decreases with maturity [24]. All semaphorins exhibit redundant structural features such as a distinctive SEMA domain, which allows both the dimerization between 2 semaphorins and the binding to receptors, 2 essential molecular events for semaphorin function. The major signal-transducing receptors are the plexins, a large family of single transmembrane-spanning cell surface receptors [25]. In addition, class 3 semaphorins bind to neuropilins (NRP1 and NRP2) which serve 
as coreceptors in concert with A-type plexins (PLXNAs), with the exception for SEMA3E, which directly binds to PLXND1.

Other transmembrane molecules are functionally coupled to semaphorin receptors, including cell adhesion molecule L1, and receptor tyrosine kinases, such as offtrack kinase (OTK), scatter factor receptors (SFRs), and vascular endothelial growth factor receptors (VEGFRs) [26]. The possibility to form receptor complexes on the membrane allows for cross talk between the different signaling pathways.

\section{Semaphorin $3 A$ and $3 F$, and Receptors}

Previous expression studies have highlighted the expression of semaphorins and neuropilins in the olfactory structures [27]. Further analysis of Nrp2-knockout (KO) mice has revealed that Nrp2 is required for axonal fasciculation/zonal segregation of vomeronasal neurons into the accessory olfactory bulb glomeruli [28]. Thus, the SEMA3F coreceptor NRP2 was then suggested to be involved in GnRH neuron migration based on in vitro and in vivo studies from our laboratory. Specifically, we found that Nrp2 is expressed by nasal axons that form the axonal scaffold followed by GnRH neurons to migrate into the nasal compartment. Accordingly, Nrp2-KO mice display defasciculation of nasal axons, with a consequent reduction in the number of hypothalamic GnRH neurons [29] and reduced fertility. The lack of a strong phenotype in the Nrp2-null mice analyzed in this study suggested that other member(s) of the class 3 semaphorin-signaling pathway might also be involved in this process and prompted us to analyze different single and compound KO mouse models. Specifically, we found that Sema3f, which is the canonical Nrp2 ligand, is dispensable for $\mathrm{GnRH}$ neuron migration, with a normal migration and number of GnRH neurons in mice lacking Sema3f. Instead, we found that Sema3a-null mice show a defective GnRH neuron migration, with clusters of cells accumulated in the nasal compartment and unable to reach the forebrain. This is due to the aberrant targeting of the cVNN which forms tangles of axons in the nasal compartment and fails to penetrate the brain [18]. Unexpectedly, but in agreement with a possible role of both Nrp1 and Nrp2 in mediating Sema3a signaling, we found that single Nrp1 mutants lacking the Sema binding domain only partially phenocopy the defects of Sema3a-null mice, whereas the combined loss of both Nrp1 and Nrp2 fully phenocopied Sema3a-null mice. These results, together with binding assays on knockout tissues, demonstrated that Sema3a via Nrp1 and Nrp2 controls targeting of the
cVNN and indirectly regulates the migration of $\mathrm{GnRH}$ neurons towards the brain. In agreement, subsequent mutational screening on IGD patients revealed mutations in the SEMA3A gene in several IGD patients $[19,21,22]$, and expression studies on human fetuses confirmed the expression of NRP1 on the cVNN [19]. Recently, it has been shown that Sema3a is highly expressed at the nasalforebrain junction level, where it may play a dual role: on the one hand, it prevents OLF/VNN from invading the forebrain, and, on the other hand, it attracts both $\mathrm{GnRH}$ neurons and the peripherin-expressing axons (cVNN according to a previous model or TN in agreement with more recent findings) that are followed by GnRH neurons [9]. This double role of Sema3a might be explained by the presence of different sets of receptors and coreceptors on each cell population: according to this working hypothesis, cVNN/TN, as well as GnRH neurons, which express Nrp1, are attracted by Sema3a, whereas OLF/ VNN, which express both Nrp1 and Nrp2, are repelled by Sema3a, preventing brain invasion [9].

In addition to differential expression of NRPs, different combinations of PLXNAs, which are obligatory transducing receptors of SEMA3A, may contribute to the diverse response to SEMA3A signaling. Thus, heterozygous PLXNA1 mutations along with mutations in SEMA3A, $N R P 1$, and NRP2 have also been reported in KS patients recently [23]. In this paper, mice with a homozygous Plxna1-null mutation showed, although with low penetrance, a reproductive phenotype, characterized by a low fertility index on postnatal day 40 and by less GnRH neuron-innervated ME [23]. The GnRH neuron defects observed in the Plxnal-KO mutant embryos are also not fully penetrant, with only one-third embryos showing defects in cVNN targeting. These observations raise the possibility that other members of the Plxnas family may additionally contribute to mediate the Sema3a effects on the targeting of the cVNN.

Further studies demonstrated that Sema3a exerts a crucial role also in adulthood by remodeling GnRH fibers that project to the ME during the estrus cycle. Specifically, brain endothelial cells released Sema3a that promotes GnRH neuron axonal sprouting through Nrp1 expressed by GnRH neuron axon terminals; in addition, the amount of Sema3a released increases dramatically during the proestrus, when GnRH is released in massive surges to sustain LH surge [30].

Overall, these pieces of evidence strongly support the existence of a dual role for Sema3a signaling in the control of reproduction via effects on prenatal GnRH neuron development and adult $\mathrm{GnRH}$ release. 


\section{Semaphorin $4 D$}

Semaphorin 4D (SEMA4D) is a membrane-bound semaphorin that is proteolytically cleaved to be able to bind PLXNB1 and Met tyrosine kinase receptor. Thus, Sema4d induces growth cone collapse by interacting with Plxnb1 [31], while it promotes angiogenesis via the combined interaction with Plxnb1 and Met [32]. In the developing olfactory system, the Sema4d receptors Plxnb1 and Plxnb2 are highly expressed [33]. Further, work from Giacobini et al. [34] demonstrated that Plxnb1 and Ncam expression colocalized in the nasal mesenchyme only at early stages of development (E12.5 but neither E14.5 nor E17.5) and that Sema4d promotes the chemomigration of immortalized GnRH neurons via the activation of Plxnb and Met signaling. Consistent with a cell-autonomous effect of Sema4d on migrating GnRH neurons in the nose, Plxnb1-null mice showed a reduced number of GnRH neurons reaching the forebrain with accumulation in the olfactory bulb region. A decrease in $\mathrm{GnRH}$ neurons in the adult brains resulted also in a decrease in $\mathrm{GnRH}$ fibers at the ME. Instead, while this study reported a normal number of GnRH neurons in adult Sema4d-KO brains, a more recent work has shown that female Sema4d-KO mice exhibit decreased levels of GnRH mRNA in the hypothalamus and reduced ovarian follicle maturation [35]. However, despite the presence of reproductive defects of both Plxnb1- and Sema4d-null mice, no human mutations in this pathway have been documented so far.

\section{Semaphorin $7 A$}

SEMA7A is the only glycosylphosphatidylinositol-anchored semaphorin, and it can act either as a membranebound protein or secreted factor after proteolytic cleavage. Although originally identified in immune cells, SEMA7A displays widespread expression and function in a wide variety of biological processes in different organs. Well-characterized biological effects of SEMA7A include bone and immune cell regulation, neuron migration, and neurite growth. These effects are mediated by PLXNC1 and integrin receptors [36]. Specifically, SEMA7A binds with equal affinity to PLXNC1, reducing the integrin-mediated cell adhesion, and to $\beta_{1}$-integrin (ITG $\beta 1$ ), to promote integrin clustering and, therefore, cell attachment.

Since Sema7a is expressed in the NP and in the olfactory bulb, a role for Sema7a was postulated. Indeed, in 2011, Messina et al. [37] demonstrated that Sema7a promotes GnRH neuron migration in the nasal compartment, whereas its expression is turned off in the MPOA. Sema7a is expressed by OLF/VN nerves during the embryonic development whilst the expression pattern of the
2 Sema7a receptors on GnRH neurons is strictly temporally and spatially regulated: in the nasal mesenchyme, GnRH neurons express $\operatorname{Itg} \beta 1$ and migrate along the Sema7a $\mathrm{a}^{+}$axonal scaffold, and instead of once crossing the brain, they begin to express Plxnc1 to cease migration. Consistently, Sema7a-null mice display defective GnRH migration and, as a consequence, a less innervated ME and dysfunctional gonads.

Further, conditional inactivation of the Sema7a receptor $\operatorname{Itg} \beta 1$ in $\mathrm{GnRH}$ neurons alters migration of $\mathrm{GnRH}$ neurons and targeting of their axons to the ME, resulting in suboptimal maturation of the GnRH system in the adult brain. Examination of fertility revealed that lack of $\beta_{1}$-integrin in GnRH neurons caused delayed pubertal onset and disruption of estrous cyclicity in female $\mathrm{GnRH}$ Itgb1 $1^{-/-}$mice, which greatly impaired reproduction. These findings demonstrated a critical role for $\beta_{1}$-integrin in GnRH neuronal function and mammalian reproduction [38].

Similarly to Sema3a, Sema7a exerts an additional function in ME plasticity, where it is expressed by tanycytes, specialized glial cells lining the third ventricle. There, Sema7a induces retraction of GnRH fiber terminals and promotes the spread of tanycyte end feet by acting through Plxnc1 and Itg $\beta 1$, respectively [39]. In agreement with the essential role exerted by Sema7A in GnRH neuron development and plasticity in mice, mutations in SEMA7A have been found both in KS and nHH patients [22].

\section{Semaphorin $3 E$}

SEMA3E has previously been shown to play different roles during development, such as neuron migration, axon guidance, and vascular system formation, as well as in cancer progression, through the interaction with different receptors and complexes. Via Plxnd1, Sema3e controls, for example, the motogenic potential of CajalRetzius cells in the developing cortex [40], and it regulates VEGF function in developmental angiogenesis via a feedback mechanism [41]. Further, Sema3e mediates axon growth interacting with Vegfr2, Plxnd1, and Nrp1 [42]. In cancer cells, SEMA3E promotes cell invasiveness and metastatic spread by transactivating the oncogenic tyrosine kinase ErbB2 that associates with PLXND1 [43], whereas it suppresses tumor cell death triggered by the PLXND1 dependence receptor in metastatic breast cancers [44].

In our recent work, we combined exome sequencing with in vitro and in vivo functional assays to identify Sema3e as an essential gene for GnRH neuron development. First, we found that the Sema3e receptor Plxnd1 is 
expressed during GnRH neuron development, with a peculiar spatial and temporal expression pattern. Specifically, we found Plxnd1 expression on blood vessels and OLF/VN axons but not on migrating GnRH neurons in the nose. Instead, when GnRH neurons enter the forebrain to scatter in the MPOA, Plxnd1 is highly expressed by $\mathrm{GnRH}$ neurons. In agreement with a possible neuroprotective role of Sema3e in the MPOA, mice lacking Plxnd1 have a reduced number of GnRH neurons in this region, and Sema3e prevents the cell death of immortalized postmigratory GnRH neurons (GT1-7 cells) [45].

Clinically, SEMA3E has been linked to the CHARGE syndrome [46], a complex syndrome whose phenotypic spectrum also includes syndromic IGD, which is in the majority of cases caused by mutations in the $\mathrm{CHD} 7$ gene. Interestingly, by further interrogating the exome of the 2 IGD-affected patients carrying heterozygous mutations of SEMA3E studied in this work, we found the coexistence of a heterozygous $C H D 7$ mutation, suggestive of a possible interaction between the 2 genetic pathways. The analysis of compound heterozygous mice for neuronal Plxnd1 and CHD7 revealed a marked reduction in both GnRH neuron ME innervation and gonadal size compared to single Chd7 heterozygous mice, used as a mouse model for the CHARGE syndrome [47]. Interestingly, recent reports have documented that Chd7, a chromatinremodeling protein that controls gene expression, regulates the expression of other members of the semaphorin family using a Chd7-deficient mouse model [48], supporting the possibility of CHD7 being an upstream regulator of semaphorin expression.

\section{Conclusions}

In conclusion, the possibility to interrogate exome sequencing data from affected individuals combined with in vitro and in vivo models aimed at validating the functional relevance of the mutated genes is likely to uncover the remaining causes of IGD. Given the complex roles of semaphorins in axon guidance, neuronal migration, as well as in neuronal plasticity and the unique development and physiology of GnRH neurons, it is not surprising if additional members of the semaphoring-plexin signaling pathways will be found implicated. Further, given the important dual role of semaphorin signaling in regulating neuronal and vascular patterning, future studies will be needed to prove their reciprocal influence in the $\mathrm{GnRH}$ system.

\section{Acknowledgments}

We would like to thank Dr. Roberta Azzarelli for critically reading this paper.

\section{Disclosure Statement}

The authors have no conflicts of interest to declare.

\section{References}

1 Herbison AE. Physiology of the gonadotropin-releasing hormone neuronal network. In: Knobil and Neill's Physiology of Reproduction. San Diego: Academic Press; 2006. pp. 1415-1482. https://doi.org/10.1016/B978012515400-0/50033-6.

2 Forni PE, Wray S. GnRH, anosmia and hypogonadotropic hypogonadism - where are we? Front Neuroendocrinol. 2015 Jan;36(2): $165-77$.

3 Wierman ME, Kiseljak-Vassiliades K, Tobet S. Gonadotropin-releasing hormone $(\mathrm{GnRH})$ neuron migration: initiation, maintenance and cessation as critical steps to ensure normal reproductive function. Front Neuroendocrinol. 2011 Jan;32(1):43-52.

4 Wray S. From nose to brain: development of gonadotrophin-releasing hormone-1 neurones. J Neuroendocrinol. 2010 Jul;22(7): $743-53$.
5 Forni PE, Taylor-Burds C, Melvin VS, Williams T, Wray S. Neural crest and ectodermal cells intermix in the nasal placode to give rise to GnRH-1 neurons, sensory neurons, and olfactory ensheathing cells. J Neurosci. 2011 May;31(18):6915-27.

6 Casoni F, Hutchins BI, Donohue D, Fornaro $\mathrm{M}$, Condie BG, Wray S. SDF and GABA interact to regulate axophilic migration of $\mathrm{GnRH}$ neurons. J Cell Sci. 2012 Nov 1;125(Pt 21): 5015-25.

7 Wray S. Development of gonadotropin-releasing hormone-1 neurons. Front Neuroendocrinol. 2002 Jul;23(3):292-316.

8 Yoshida K, Tobet SA, Crandall JE, Jimenez TP, Schwarting GA. The migration of luteinizing hormone-releasing hormone neurons in the developing rat is associated with a transient, caudal projection of the vomeronasal nerve. J Neurosci. 1995 Dec;15(12):7769-77.
9 Taroc EZ, Prasad A, Lin JM, Forni PE. The terminal nerve plays a prominent role in GnRH-1 neuronal migration independent from proper olfactory and vomeronasal connections to the olfactory bulbs. Biol Open. 2017 Oct;6(10): 1552-68.

10 Casoni F, Malone SA, Belle M, Luzzati F, Collier F, Allet C, et al. Development of the neurons controlling fertility in humans: new insights from 3D imaging and transparent fetal brains. Development. 2016 Nov; 143(21): 3969-81.

11 Balasubramanian R, Crowley WF Jr. Reproductive endocrine phenotypes relating to CHD7 mutations in humans. Am J Med Genet C Semin Med Genet. 2017 Dec;175(4):507-15.

12 Stamou MI, Georgopoulos NA. Kallmann syndrome: phenotype and genotype of hypogonadotropic hypogonadism. Metabolism. 2018 Sep;86:124-34. 
13 Boehm U, Bouloux PM, Dattani MT, de Roux N, Dodé C, Dunkel L, et al. Expert consensus document: European Consensus Statement on congenital hypogonadotropic hypogonadism - pathogenesis, diagnosis and treatment. Nat Rev Endocrinol. 2015 Sep;11(9):547-64.

$14 \mathrm{Kim}$ SH. Congenital Hypogonadotropic Hypogonadism and Kallmann Syndrome: Past, Present, and Future. Endocrinol Metab (Seoul). 2015 Dec;30(4):456-66.

15 Sykiotis GP, Plummer L, Hughes VA, Au M, Durrani S, Nayak-Young S, et al. Oligogenic basis of isolated gonadotropin-releasing hormone deficiency. Proc Natl Acad Sci USA. 2010 Aug;107(34):15140-4.

16 Marino M, Moriondo V, Vighi E, Pignatti E, Simoni M. Central hypogonadotropic hypogonadism: genetic complexity of a complex disease. Int J Endocrinol. 2014;2014:649154.

17 Stamou MI, Cox KH, Crowley WF Jr. Discovering Genes Essential to the Hypothalamic Regulation of Human Reproduction Using a Human Disease Model: Adjusting to Life in the "-Omics" Era. Endocr Rev. 2015 Dec; 36(6):603-21.

18 Cariboni A, Davidson K, Rakic S, Maggi R, Parnavelas JG, Ruhrberg C. Defective gonadotropin-releasing hormone neuron migration in mice lacking SEMA3A signalling through NRP1 and NRP2: implications for the aetiology of hypogonadotropic hypogonadism. Hum Mol Genet. 2011 Jan;20(2):336-44.

19 Hanchate NK, Giacobini P, Lhuillier P, Parkash J, Espy C, Fouveaut C, et al. SEMA3A, a gene involved in axonal pathfinding, is mutated in patients with Kallmann syndrome. PLoS Genet. 2012 Aug;8(8):e1002896.

20 Cariboni A, André V, Chauvet S, Cassatella D, Davidson K, Caramello A, et al. Dysfunctional SEMA3E signaling underlies gonadotropin-releasing hormone neuron deficiency in Kallmann syndrome. J Clin Invest. 2015 Jun; 125(6):2413-28.

21 Young J, Metay C, Bouligand J, Tou B, Francou B, Maione L, et al. SEMA3A deletion in a family with Kallmann syndrome validates the role of semaphorin $3 \mathrm{~A}$ in human puberty and olfactory system development. Hum Reprod. 2012 May;27(5):1460-5.

22 Känsäkoski J, Fagerholm R, Laitinen EM, Vaaralahti K, Hackman P, Pitteloud N, et al. Mutation screening of SEMA3A and SEMA7A in patients with congenital hypogonadotropic hypogonadism. Pediatr Res. 2014 May;75(5):641-4.

23 Marcos S, Monnier C, Rovira X, Fouveaut C, Pitteloud N, Ango F, et al. Defective signaling through plexin-A1 compromises the development of the peripheral olfactory system and neuroendocrine reproductive axis in mice. Hum Mol Genet. 2017 Jun;26(11):2006-17.

24 Yazdani U, Terman JR. The semaphorins. Genome Biol. 2006;7(3):211.
25 Tamagnone L, Artigiani S, Chen H, He Z, Ming GI, Song $\mathrm{H}$, et al. Plexins are a large family of receptors for transmembrane, secreted, and GPI-anchored semaphorins in vertebrates. Cell. 1999 Oct;99(1):71-80.

26 Tamagnone L, Comoglio PM. To move or not to move? Semaphorin signalling in cell migration. EMBO Rep. 2004 Apr;5(4):356-61.

27 Giger RJ, Wolfer DP, De Wit GM, Verhaagen J. Anatomy of rat semaphorin III/collapsin-1 mRNA expression and relationship to developing nerve tracts during neuroembryogenesis. J Comp Neurol. 1996 Nov;375(3):37892.

28 Cloutier JF, Giger RJ, Koentges G, Dulac C, Kolodkin AL, Ginty DD. Neuropilin-2 mediates axonal fasciculation, zonal segregation, but not axonal convergence, of primary accessory olfactory neurons. Neuron. 2002 Mar; 33(6):877-92.

29 Cariboni A, Hickok J, Rakic S, Andrews W, Maggi R, Tischkau S, et al. Neuropilins and their ligands are important in the migration of gonadotropin-releasing hormone neurons. J Neurosci. 2007 Feb;27(9):2387-95.

30 Giacobini P, Parkash J, Campagne C, Messina A, Casoni F, Vanacker C, et al. Brain endothelial cells control fertility through ovarian-steroid-dependent release of semaphorin 3A. PLoS Biol. 2014 Mar;12(3):e1001808.

31 Ito Y, Oinuma I, Katoh H, Kaibuchi K, Negishi M. Sema4D/plexin-B1 activates GSK-3 $\beta$ through R-Ras GAP activity, inducing growth cone collapse. EMBO Rep. 2006 Jul;7(7): 704-9.

32 Conrotto P, Valdembri D, Corso S, Serini G, Tamagnone L, Comoglio PM, et al. Sema4D induces angiogenesis through Met recruitment by Plexin B1. Blood. 2005 Jun;105(11): 4321-9.

33 Perälä N, Sariola H, Immonen T. More than nervous: the emerging roles of plexins. Differentiation. 2012 Jan;83(1):77-91.

34 Giacobini P, Messina A, Morello F, Ferraris N, Corso S, Penachioni J, et al. Semaphorin $4 \mathrm{D}$ regulates gonadotropin hormone-releasing hormone-1 neuronal migration through PlexinB1-Met complex. J Cell Biol. 2008 Nov; 183(3):555-66.

35 Dacquin R, Domenget C, Kumanogoh A, Kikutani H, Jurdic P, Machuca-Gayet I. Control of bone resorption by semaphorin $4 \mathrm{D}$ is dependent on ovarian function. PLoS One. 2011;6(10):e26627.

36 Jongbloets BC, Ramakers GM, Pasterkamp RJ. Semaphorin7A and its receptors: pleiotropic regulators of immune cell function, bone homeostasis, and neural development. Semin Cell Dev Biol. 2013 Mar;24(3):129-38.
37 Messina A, Ferraris N, Wray S, Cagnoni G, Donohue DE, Casoni F, et al. Dysregulation of Semaphorin7A/ $\beta 1$-integrin signaling leads to defective GnRH-1 cell migration, abnormal gonadal development and altered fertility. Hum Mol Genet. 2011 Dec;20(24):475974.

38 Parkash J, Cimino I, Ferraris N, Casoni F, Wray S, Cappy H, et al. Suppression of $\beta 1$ integrin in gonadotropin-releasing hormone cells disrupts migration and axonal extension resulting in severe reproductive alterations. J Neurosci. 2012 Nov;32(47):16992-7002.

39 Parkash J, Messina A, Langlet F, Cimino I, Loyens A, Mazur D, et al. Semaphorin7A regulates neuroglial plasticity in the adult hypothalamic median eminence. Nat Commun. 2015 Feb;6(1):6385.

40 Bribián A, Nocentini S, Llorens F, Gil V, Mire E, Reginensi D, et al. Sema3E/PlexinD1 regulates the migration of hem-derived CajalRetzius cells in developing cerebral cortex. Nat Commun. 2014 Jun;5(May):4265.

41 Kim J, Oh WJ, Gaiano N, Yoshida Y, Gu C. Semaphorin 3E-Plexin-D1 signaling regulates VEGF function in developmental angiogenesis via a feedback mechanism. Genes Dev. 2011 Jul;25(13):1399-411.

42 Bellon A, Luchino J, Haigh K, Rougon G, Haigh J, Chauvet S, et al. VEGFR2 (KDR/ Flk1) signaling mediates axon growth in response to semaphorin $3 \mathrm{E}$ in the developing brain. Neuron. 2010 Apr;66(2):205-19.

43 Casazza A, Kigel B, Maione F, Capparuccia L, Kessler O, Giraudo E, et al. Tumour growth inhibition and anti-metastatic activity of a mutated furin-resistant Semaphorin 3E isoform. EMBO Mol Med. 2012 Mar;4(3):23450.

44 Luchino J, Hocine M, Amoureux MC, Gibert B, Bernet A, Royet A, et al. Semaphorin 3E suppresses tumor cell death triggered by the plexin D1 dependence receptor in metastatic breast cancers. Cancer Cell. 2013 Nov;24(5): 673-85.

45 Mellon PL, Windle JJ, Goldsmith PC, Padula CA, Roberts JL, Weiner RI. Immortalization of hypothalamic GnRH neurons by genetically targeted tumorigenesis. Neuron. 1990 Jul;5(1):1-10

46 Lalani SR, Safiullah AM, Molinari LM, Fernbach SD, Martin DM, Belmont JW. SEMA3E mutation in a patient with CHARGE syndrome. J Med Genet. 2004 Jul;41(7):e94.

47 Randall V, McCue K, Roberts C, Kyriakopoulou V, Beddow S, Barrett AN, et al. Great vessel development requires biallelic expression of Chd7 and Tbx1 in pharyngeal ectoderm in mice. J Clin Invest. 2009 Nov;119(11):330110.

48 Schulz Y, Wehner P, Opitz L, Salinas-Riester G, Bongers EM, van Ravenswaaij-Arts CM, et al. CHD7, the gene mutated in CHARGE syndrome, regulates genes involved in neural crest cell guidance. Hum Genet. 2014 Aug; 133(8):997-1009. 